\title{
In silico Analysis on the Possible Role of Mitochondria in Ferroptosis
}

\author{
Tamás Lőrincz ${ }^{1}$ András Szarka1* \\ 1 Department of Applied Biotechnology and Food Science, Faculty of Chemical Technology and Biotechnology, \\ Budapest University of Technology and Economics, H-1521 Budapest, P.O.B. 91, Hungary \\ *Corresponding author, e-mail: szarka@mail.bme.hu
}

Received: 24 May 2018, Accepted: 28 June 2018, Published online: 07 August 2018

\begin{abstract}
The lipid peroxide scavenger enzyme glutathione peroxidase 4 (GPX4) and its cofactor glutathione play a crucial role in the recently described programmed cell death, ferroptosis. Since mitochondria are the major sources of reactive oxygen species in mammalian cells the linkage between mitochondria, mitochondrial ROS generation and ferroptosis emerged quite early. As a result of alternative splicing human GPX4 has three different isoforms: long form (IGPX4), short form (sGPX4) and nuclear form (nGPX4) of which sGPX4 was found essential. To clarify the potential role of mitochondria in ferroptosis the localization of both the long and short versions of GPX4 and the only verified glutathione transport protein SLC25A11 was investigated by different in silico tools. Targeting of IGPX4 and SGPX4 to multiple organelles is possible as a number of rule- and neural network-based algorithms showed concordant results to specific pathways. Hence the mitochondrial localization of both isoforms on the base of in silico prediction is possible. Since sGPX4 was found in multiple organelles and non-canonical import pathways are presumable we also evaluated the previously used in silico methods in predicting the localization of a chimeric signal containing peptide CYP2B1 as well as a solely mitochondrial targeted CYP27A1. Our in silico results showed that only the CELLO prediction tool ranked mitochondrial import with meaningful possibility based on neighboring sequence composition. Summarily the possible mitochondrial localization of both long and short isoforms of GPX4 and the glutathione transporter SLC25A11 support the assumption that mitochondria play an important role in the pathways leading to ferroptosis.
\end{abstract}

\section{Keywords}

mitochondria, ferroptosis, GPX4, glutathione, in silico, subcellular localization

\section{Introduction}

The water-soluble tripeptide glutathione (GSH) is exclusively synthesized in the cytosol where it can be found in millimolar range [1-3]. As a cofactor of glutathione peroxidase it quenches hydrogen-peroxide and repairs lipid oxidation, scavenges reactive nitrogen species (RNS), the ratio of its reduced/oxidized form (GSH/GSSG) serves as a sensitive factor of cellular redox state, hence it mediates redox signaling, furthermore it conjugates with drugs and xenobiotics under the catalysis of glutathione S-transferases (GSTs) [4]. Looking at this list of important functions it is not surprising that extensive GSH depletion was involved in different cell deaths such as apoptosis, necroptosis and autophagy [5-8]. Beyond these cell death types GSH and GSH depletion plays a crucial role in a recently described novel programmed cell death, ferroptosis. Ferroptosis has been induced (and described) for the first time by the addition of the small molecule erastin to oncogenic RASmutant cell lines $[9,10]$. Erastin treatment causes a marked depletion in the GSH content by the inhibition of the cystine/glutamate antiporter. GSH depletion by erastin has clearly been necessary for its lethality since ferroptotic cell death could be prevented by the supplementation of the culture medium with GSH or N-acetylcysteine [11]. The further important feature of ferroptotic cell death was the increased levels of lipid hydroperoxides [11]. The generation of lipid ROS and the induction of ferroptosis could also be observed by the direct inhibition or by the knockdown of GPX4 [11]. On the base of these observations ferroptosis inducers (FIN) have been classified into two classes: type I agents (e.g. erastin) that involve GSH depletion and type II agents (e.g. RSL3, RSL5, FIN56, FINO2) that trigger ferroptosis through inhibition of GPX4 [12]. RSL3 directly 
inactivates GPX4, FIN56 accelerates the specific degradation of GPX4 [13], while the organic peroxide FINO2 initiates lipid peroxidation and iron oxidation [14]. Erastin or glutamate inactivates GPX4 indirectly through the inhibition of the cystine/glutamate antiporter, causing subsequent cystine and GSH depletion and reduced GPX4 activity $[9,10]$. GPX4 is a GSH dependent seleno-protein with the function to eliminate lipid peroxides. Human GPX4 is a functional monomer and is said to have three isoforms produced by alternative splicing: 1GPX4 (long form, also called mitochondrial), sGPX4 (short form, also called cytosolic) and nGPX4 (nuclear form). 1GPX4 is synthetized from the first start codon and thus, as opposed to sGPX4 contains an extra 27 amino acid sequence at the N-terminal, which was described to participate in the mitochondrial import of 1GPX4 [15]. Both start codons are located on the first exon of the GPX4 gene and it is still debated whether the expression is regulated on a transcriptional or translational level [16].

In an experiment with transgenic mice where either IGPX4 or sGPX4 was overexpressed, it was found that sGPX4 - albeit not having a mitochondrial targeting presequence - was found to localize in mitochondria and in other organelles as well of somatic tissue [17]. It was also found that sGPX4 but not IGPX4 was able to rescue the lethal knockout of the GPX4 gene and it provided protection against apoptosis induced by diquat [17]. On the behalf of the other isoform IGPX4 was found to be mainly expressed in testis with a role in male fertility [17].

nGPX4 is synthetized from an alternative exon which codes for an arginine rich nuclear insertion sequence showing high similarity to other known import signals [18]. As it was found, nGPX4 is primarily expressed during late spermatogenesis where it is the only seleno-protein present in the spermatid nuclei and its' reduction in expression resulted in aberrant chromatin condensation and elevation in oxidative damage to DNA [18]. However as the knockout of nGPX4 in mice - as opposed to the deletion of the full GPX4 gene which results in early embryonic lethalitydid not result in infertility or any major lesions [19] its' biological significance is still investigated.

Mitochondria lay in the centre of cellular oxidative metabolism and are the major intracellular ROS source of most human cell types. Mitochondrial ROS are continuously generated as a by-product of oxidative phosphorylation and linked to cellular degeneration. ROS derived from mitochondria may lead to oxidative damage of biomolecules that promote cell death [20]. Thus the linkage between mitochondria, mitochondrial ROS generation and ferroptosis has emerged quite early. This assumption was strengthened by the knockdown of VDAC3 that suppressed both the erastin and RSL5-induced cell death [21]. However in a later study - on the contrary of the mitochondrial localization of the two erastin targets, VDAC2 and VDAC3 - no increase in MitoSOX-sensitive mitochondrial ROS production could be observed due to erastin-treatment [10]. Furthermore, similar ROS accumulation and cell death could be provoked by erastin and RSL3 in Rho0 cells lacking a functional mitochondrial electron transfer chain (ETC), as in the wild type (with functional mitochondrial ETC). These later observations disputed the role of mitochondria in the mechanism of ferroptosis [10]. However in the same study in an attempt to identify the genetic basis of erastin sensitivity a set of proteins localized in mitochondria were targeted through RNA silencing and among others, two genes (acyl-CoA synthetase family member 2 - ACSF2 - and citrate synthase - CS) involved in mitochondrial fatty acid metabolism were identified [10]. In a very recent study lipid peroxidation, enhanced mitochondrial fragmentation, loss of mitochondrial membrane potential, and reduced mitochondrial respiration was induced by RSL3. Beyond the well-known ferroptosis inhibitors, such as deferoxamine, ferrostatin-1 and liproxstatin-1 the knockout of Bid and the BID inhibitor BI-6c9 could protect the cells against the toxicity of RSL3. The mitochondria-targeted ROS scavenger mitoquinone - despite the significant loss of GPX4 activity and the associated significant lipid peroxidation could also preserve the mitochondrial function, and cell viability [22]. Specific ferroptosis inhibitors - ferrostatin-1 and liproxstatin-1 - are known to alleviate cell demise by blockage of upstream processes to ROS production and lipid peroxidation. As of the arylalkylamine structure of ferrostain-1 a potential direct antioxidant function through reductive mechanism was suggested by Skouta et al. [23], by which it could inhibit oxidative damage to membrane lipids and thus cell death. All these observations highlighted the potential role of mitochondria (their function and integrity) in the mechanism of ferroptosis. The role of mitochondria in the generation of ROS is indisputable. However, the role of GPX4 in the elimination of lipid ROS in mitochondria has not been fully elucidated yet.

To clarify this role the localization of both the long and short version of GPX4 and the only verified GSH transport protein SLC25A11 [24] was investigated by different in silico tools. 


\section{Materials and methods}

\subsection{Protein sequences}

The sequences of both human GPX4 isoforms (Uniprot identifiers: 1GPX4: P36969; sGPX4: P36969-2), human CYP27A1 (Uniprot identifier: Q02318), rat CYP2B1 (Uniprot identifier: P00176) and human 2-oxoglutarate/ malate carrier protein SLC25A11 (Uniprot identifier: Q6IBH0) were retrieved from the Uniprot database (http:// www.uniprot.org/).

\subsection{Prediction tools}

The subcellular localization of proteins (GPX4, CYP27A1, CYP2B1 and SLC25A11) was predicted by an in silico analysis using eight different prediction software:

- Target P: http://www.cbs.dtu.dk/services/TargetP/ [25]

- Mitoprot II: http://ihg.gsf.de/ihg/mitoprot.html [26]

- Predotar: https://urgi.versailles.inra.fr/predotar/predotar.html [27]

- Psort II: http://psort.hgc.jp/form2.html [28]

- iPSORT: http://ipsort.hgc.jp/index.html [29]

- MultiLoc/TargetLoc: http://abi.inf.uni-tuebingen.de/ Services/MultiLoc/ [30]

- ngLOC: http:/genome.unmc.edu/ngLOC/index.html [31]

- YLoc: http://abi.inf.uni-tuebingen.de/Services/ YLoc/webloc.cgi [32]

- CELLO v2.5: http://cello.life.nctu.edu.tw/ [33]

The analyses were done in May of 2018.

\section{Results and discussion}

Mitochondria play a central role in cellular bioenergetics, ROS homeostasis, and in various cell death pathways as in apoptosis and necroptosis $[34,35]$. Therefore, it is not surprising that the role of mitochondria is continuously investigated in a cell death pathway which has features such as oxidative damage to lipid structures, the involvement of glutathione dependent antioxidant enzymes and the biosynthesis of specific lipid moieties.

As previous results indicated the differential localization of GPX4 isoforms had a profound impact on survivability and cell function, thus we sought to investigate the subcellular localization of the two physiologically most relevant isoforms 1GPX4 and sGPX4 with the aid of in silico techniques.

1GPX4 differs from sGPX4 by the N-terminal presequence which according to our results using the Mitoprot algorithm shows strong mitochondrial localization (Table 1). Mitoprot uses a rule-based method to identify N-terminal mitochondrial import signal sequences [26], while Predotar and TargetP use a trained neural network [25, 27].

It is interesting to note that the neural network based algorithms rank endoplasmic reticulum import pathway highly: TargetP calculated some degree of mitochondrial localization, but processing of the 1GPX4 peptide through the secretory pathway (SP) was considered to be more than 3 times as probable while ER localization calculated by Predotar was almost as probable as mitochondrial (Table 1). This result was also supported by PSORT II and CELLO two rule-based algorithms as extracellular localization was calculated as most probable based on the $\mathrm{N}$-peptide and neighboring sequence composition (Table 1). Interestingly the N-terminal signal was identified by an extension of PSORT II named iPSORT but based on the average net charge and pattern it was calculated as non-mitochondrial (data not shown).

Other algorithms such as ngLOC and CELLO also ranked mitochondrial localization to the top with possible cytoplasmic localization (Table 1). These are in accordance with the results demonstrating that the N-terminal presequence can function as a mitochondrial import signal [15].

The subcellular localization of sGPX4 was predicted. The absence of the N-terminal presequence diminished the

Table 1 in silico prediction of the subcellular localization of the long isoform of GPX4 (1GPX) using different algorithms. (ER: endoplasmic reticulum, ER-SP: endoplasmic reticulum - secretory pathway, mito: mitochondrion, extracell: extracellular space, cyto: cytoplasm, nuc: nucleus).

\begin{tabular}{|c|c|c|c|c|c|c|}
\hline \multirow[b]{3}{*}{ TargetP } & \multicolumn{6}{|c|}{ long isoform of GPX4 (1GPX4) } \\
\hline & \multicolumn{2}{|c|}{ 1st rank } & \multicolumn{2}{|c|}{ 2nd rank } & \multicolumn{2}{|c|}{3 rd rank } \\
\hline & 0.742 & ER-SP & 0.292 & mito & 0.013 & other \\
\hline Predotar & 0.43 & mito & 0.37 & other & 0.35 & ER \\
\hline PSORT II & 66.7 & extracell & 11.1 & cyto & 11.1 & ER \\
\hline ngLOC & 34.6 & mito & 34.5 & cyto & 16.6 & nuc \\
\hline CELLO & 1.55 & extracell & 1.44 & mito & 1.146 & cyto \\
\hline MitoProt & \multicolumn{6}{|c|}{0.869 mito, no predictable cleavage site } \\
\hline iPSORT & \multicolumn{6}{|c|}{ non-mitochondrial signal peptide, non-mitochondrial localization } \\
\hline
\end{tabular}


probability of import through the endoplasmic reticulum pathway as calculated by both the neural network-based algorithms TargetP, Predotar and rule-based algorithms CELLO, PSORT II (Table 2).

sGPX4 is considered to be mainly the cytoplasmic isoform which was supported by PSORT II and CELLO the latter algorithm finding every feature of the peptide as cytoplasmic and both algorithms showing low probability of mitochondrial localization (Table 2).

However, Mitoprot and ngLOC algorithms show significant mitochondrial localization of sGPX4, with ngLOC showing mitochondrial localization being as probable as cytoplasmic (Table 2). Moreover, the multi-localized confidence score (MLCS, if MLCS $\geq 60$ multiple localization is calculated) predicted by ngLOC for sGPX4 was 69.74 while for 1GPX4 was 69.04.

Based on these results targeting of 1GPX4 and sGPX4 to multiple organelles is possible as a number of rule- and neural network-based algorithms showed concordant results to specific pathways. As the overexpression of human sGPX4 in transgenic mice showed that the elevated protein levels can be found in mitochondria [17], a non-canonical mitochondrial import pathway of sGPX4 is presumable.

Dual targeting of peptide sequences is possible through different mechanisms. It was shown in the example of GSTa4-4 (Glutathione S-transferase alpha 4-4) with a C-terminal mitochondrial targeting sequence which functions as a switch between mitochondrial and cytosolic localization dependent on the C-terminal phosphorylation of specific serine and threonine residues [36]. In this case oxidative stress and kinase activity dependent hyper-phosphorylation leads to chaperon (Hsp70) binding which inhibits cytosolic homodimer formation and thus retains an import-competent form [36]. However this targeting strategy of sGPX4 is not so possible as it was shown through X-ray crystallography that in physiological conditions it is a functional monomer [37].

A similar mechanism of bimodal protein targeting to either endoplasmic reticulum or mitochondria was described for different CYP (Cytochrome P450 oxidase) isoforms lacking an N-terminal mitochondrial targeting sequence [38]. Dually targeted CYPs' (e.g. CYP1A1, 2B1, 2E1 and 2D6 [39]) are primarily processed through the SRP (signal recognition particle) pathway involving SRP-binding to the hydrophobic N-terminal of the nascent polipeptid and delivery to the ER through the translocon complex. However in this case the N-terminal sequence is a chimeric signal: the ER targeting region is flanked by a cryptic mitochondrial targeting sequence which can be activated by either proteolytic cleavage (family I., e.g. CYP1A1) or phosphorylation (family II., e.g. CYP2B1, 2E1, 2D6) [39, 40]. The structure of the two families of chimeric signals show a slight difference: both contain the N-terminal ER targeting sequence which is followed by the mitochondrial signal and a proline rich region, but family I signals contain a proteolytic cleavage site just between the ER and mitochondrial targeting sequences, while family II chimeric sequences contain a downstream phosphorylation site (usually a serine residue) [39].

The mechanism of bimodal targeting is well studied at the level of specific CYP proteins where several sequence regions responsible of targeting have been identified.

Since the N-terminal presequence lacking sGPX4 was found in multiple organelles and non-canonical import pathways are presumable we evaluated by the previously used in silico methods the localization of a chimeric signal containing peptide CYP2B1 as well as a solely mitochondrial targeted CYP27A1 (Table 3).

CYP27A1 contains a canonical N-terminal cleavable mitochondria targeting sequence [41] which was detected

Table 2 in silico prediction of the subcellular localization of the short isoform of GPX4 (sGPX) using different algorithms. (ER: endoplasmic reticulum, ER-SP: endoplasmic reticulum - secretory pathway, mito: mitochondrion, extracell: extracellular space, cyto: cytoplasm, nuc: nucleus, cytoskel: cytoskeleton).

\begin{tabular}{lcccccc}
\hline \multicolumn{7}{c}{ short isoform of GPX4 (sGPX4) } \\
\cline { 2 - 7 } & \multicolumn{2}{c}{ 1st rank } & \multicolumn{2}{c}{ 2nd rank } & \multicolumn{2}{c}{ 3rd rank } \\
\hline TargetP & 0.797 & other & 0.215 & mito & 0.048 & ER-SP \\
Predotar & 0.98 & other & 0.02 & mito & 0.01 & ER \\
PSORT II & 65.2 & cyto & 13.0 & nuc & 13.0 & cytoskel \\
ngLOC & 34.9 & cyto & 34.9 & mito & 18.0 & nuc \\
CELLO & 3.29 & cyto & 0.50 & nuc & 0.50 & mito \\
\hline MitoProt & \multicolumn{7}{c}{0.5428 mito, no predictable cleavage site } & \\
iPSORT & \multicolumn{7}{c}{ no signal peptide, non-mitochondrial localization } \\
\hline
\end{tabular}


Table 3 in silico prediction of the subcellular localization of two different CYP450 enzymes with either canonical mitochondrial targeting (CYP27A1) or a non-canonical bimodal ER-mitochondrial chimeric signal (CYP2B1) using different algorithms. (ER: endoplasmic reticulum, ER-SP: endoplasmic reticulum - secretory pathway, mito: mitochondrion, cyto: cytoplasm, nuc: nucleus, lys: lysosome, perox: peroxisome, plasm: plasma membrane).

\begin{tabular}{|c|c|c|c|c|c|c|c|c|c|c|c|c|}
\hline \multirow[b]{3}{*}{ TargetP } & \multicolumn{6}{|c|}{ CYP27A1 } & \multicolumn{6}{|c|}{ CYP2B1 } \\
\hline & \multicolumn{2}{|c|}{ 1st rank } & \multicolumn{2}{|c|}{ 2nd rank } & \multicolumn{2}{|c|}{ 3rd rank } & \multicolumn{2}{|c|}{ 1st rank } & \multicolumn{2}{|c|}{ 2nd rank } & \multicolumn{2}{|c|}{ 3rd rank } \\
\hline & 0.931 & mito & 0.09 & other & 0.056 & ER-SP & 0.946 & ER-SP & 0.100 & mito & 0.017 & other \\
\hline Predotar & 0.90 & mito & 0.09 & other & 0.02 & ER-SP & 0.99 & ER & 0.01 & other & 0.00 & mito \\
\hline PSORT II & $47.8 \%$ & cyto & $21.7 \%$ & mito & $21.7 \%$ & nuc & 44.4 & ER & 22.2 & lys & 11.1 & mito \\
\hline ngLOC & 53.23 & mito & 8.04 & nuc & 5.84 & cyto & 65.38 & ER & 5.62 & plasm & 4.89 & nuc \\
\hline CELLO & 3.953 & mito & 0.448 & perox & 0.292 & cyto & 1.593 & plasm & 0.90 & mito & 0.59 & lys \\
\hline
\end{tabular}

MitoProt 0.997 mito, cleavage site present 0.074 mito, cleavage site present

iPSORT mitochondrial targeting peptide, mitochondrial loc.

non-mito signal peptide, non-mitochondrial loc.

by MitoProt and iPSORT as well (Table 3 CYP27A1). Nearly all algorithms (TargetP, MitoProt, Predotar, ngLOC and CELLO) predicted mitochondrial localization with high probability with the exception of PSORT II which ranked cytoplasmic localization highest with mitochondrial being second highest (Table 3 CYP27A1). For CYP27A1 an MLCS score of 33.6 was calculated by ngLOC. These results indicated that the in silico methods could predict the localization of proteins with canonical mitochondrial signals reliably.

In the case of CYP2B1 containing chimeric signals - comprising an N-terminal ER import signal, a cryptic mitochondrial targeting sequence and a phosphorylation site - the prediction efficiency of in silico algorithms was diverse (Table 3 CYP2B1). Most algorithms (TargetP, Predotar, PSORT II and ngLOC) predicted ER localization while CELLO differs by ranking plasma membrane localization highest (Table 3 CYP2B1). MitoProt detected an N-terminal proteolytic cleavage site while iPSORT found a targeting sequence based on the average hydropathy, but was termed non-mitochondrial because of its' average net charge and pattern (Table 3 CYP2B1). For CYP2B1 an
MLCS score of 28.6 was calculated by ngLOC which suggests that the algorithm was not able to detect the chimeric signal. Interestingly only CELLO ranked mitochondrial import with meaningful possibility based on the neighboring sequence composition.

As widely demonstrated in the literature ferroptosis could be induced by the inhibition or knock down of GPX4 and also by the inhibition of the transport of its substrate, GSH [11]. The GSH supply plays critical role in enzyme activity and in the induction of ferroptosis. Thus beyond the localization of GPX4 the localization of the GSH transporter protein SLC25A11 (2-oxoglutarate carrier:OGC) was also investigated by the aforementioned prediction tools. The prediction tool ngLOC that also uses gene ontology analysis [31] gave high mitochondrial localization score and other three prediction tools (TargetP, MitoProt and CELLO) give moderate scores (Table 4). PSORT II gave a low probability while Predotar and iPSORT practically gave no probability of the mitochondrial localization of SLC25A11 (Table 4). Interestingly Mitoprot found a mitochondrial cleavage site within the protein, but iPSORT could not detect any signal peptide, accordingly it predicted

Table 4 in silico prediction of the subcellular localization of SLC25A11 using different algorithms. (ER: endoplasmic reticulum, ER-SP: endoplasmic reticulum - secretory pathway, mito: mitochondrion, extracell: extracellular space, cyto: cytoplasm, nuc: nucleus, plasm: plasma membrane, perox: peroxisome).

\begin{tabular}{|c|c|c|c|c|c|c|}
\hline \multirow[b]{3}{*}{ TargetP } & \multicolumn{6}{|c|}{ SLC25A11 } \\
\hline & \multicolumn{2}{|c|}{ 1st rank } & \multicolumn{2}{|c|}{ 2nd rank } & \multicolumn{2}{|c|}{ 3rd rank } \\
\hline & 0.812 & other & 0.164 & mito & 0.079 & ER-SP \\
\hline Predotar & 0.97 & other & 0.02 & ER & 0.00 & mito \\
\hline PSORT II & 65.2 & cyto & 8.7 & nuc & 8.7 & mito \\
\hline ngLOC & 61.5 & mito & 5.9 & nuc & 5.8 & extracell \\
\hline CELLO & 2.51 & plasm & 1.08 & mito & 0.27 & perox \\
\hline MitoProt & \multicolumn{6}{|c|}{0.116 mito, cleavage site present } \\
\hline iPSORT & \multicolumn{6}{|c|}{ no signal peptide, non-mitochondrial localization } \\
\hline
\end{tabular}


non-mitochondrial localization (Table 4). At this point it is worth to have a look at the presumed mitochondrial GSH transporters. Although several previous studies have suggested that the mitochondrial dicarboxylate (DIC) and 2-oxoglutarate carriers were responsible for glutathione uptake [42-44] no transport of GSH by DIC and OGC was observed by using membrane vesicles of Lactococcus lactis overexpressing these carriers [45]. However it should be noted that NSC34 neuronal cell line that stably overexpress OGC shows an increased mitochondrial GSH level [46]. This assumption was strengthened by a very recent study in which hepatocellular carcinoma cells (HCC) and liver explants from patients with hepatocellular carcinoma exhibit selective OGC upregulation, that contributes to mGSH maintenance [24] which contributes to cell death resistance by antagonizing mitochondrial outer membrane (MOM). Furthermore the silencing of OGC but not DIC reduces mitochondrial GSH (mGSH) levels and sensitized HCC cells to hypoxia-induced ROS generation. The silencing of OGC also resulted in cell death and impaired cell growth in three-dimensional multicellular HCC spheroids that were reversible upon mGSH replenishment [24] which contributes to cell death resistance by antagonizing mitochondrial outer membrane. All these in silico, in vitro and in vivo observations strengthen and underline the role of SLC25A11 in the mitochondrial GSH transport and the co-operation of SLC25A11 and support GPX4 mitochondrial localization in the avoidance of ferroptosis.

\section{Conclusion}

Ferroptosis is a novel cell death type that is induced by lipid peroxides [10]. Since the vast majority of ROS are generated by the mitochondrial electron transfer chain the involvement of mitochondria, mitochondrial ROS generation and ROS elimination in the ferroptotic pathway was assumed. The only key protein that was recognized up to date to have a role in ferroptosis is the lipid peroxide scavenger GPX4. The direct inhibition and the knock down of

\section{References}

[1] Meister, A. "Glutathione metabolism and its selective modification", Journal of Biological Chemistry, 263(33), pp. 17205-17208, 1988.

[2] Lörincz, T., Szarka, A. "The determination of hepatic glutathione at tissue and subcellular level", Journal of Pharmacological and Toxicological Methods, 88(1), pp. 32-39, 2017. https://doi.org/10.1016/j.vascn.2017.05.004
GPX4 or the depletion of its substrate GSH by the inhibition of GSH transport system lead to ferroptosis. Thus the subcellular localization of physiological relevant GPX4 isoforms (short, long and nuclear) and the localization of the only verified GSH transport protein SLC25A11 has been investigated by in silico tools. Special attention was paid to the possible mitochondrial context by in silico tools above all regarding mitochondrial localization. Number of ruleand neural network-based algorithms showed concordant results to specific pathways for both the long and short isoform of GPX4. Thus their targeting to multiple organelles is possible. Non-canonical import pathways are presumable for the short form therefore we also evaluated by using the same in silico methods the possible localization of a chimeric signal peptide present in CYP2B1 as well as a solely mitochondrial targeted CYP27A1. The chimeric signals of CYP2B1 includes an N-terminal ER import signal, a cryptic mitochondrial targeting sequence and a phosphorylation site. The prediction efficiency of in silico algorithms in this case was diverse. Only CELLO could rank mitochondrial import with meaningful possibility based on the neighboring sequence composition. For the GSH transporter SLC25A11 two of seven prediction tool gave high, three gave moderate mitochondrial localization possibility. These results support the assumption that SLC25A11 as the GSH supplier of GPX4 take part in the protection of mitochondria against lipid peroxides. Summarily the possible co-operation of SLC25A11 and GPX4 in the mitochondrion may play a role in the regulation of ferroptosis and strengthen the involvement of mitochondria in the ferroptotic pathway.

\section{Acknowledgement}

This work was financially supported by the National Scientific Research Fund grant (OTKA 123752), by the BME-Biotechnology FIKP grant of EMMI, by ÚNKP-173-III New National Excellence Program of the Ministry of Human Capacities and MedinProt Protein Excellence foundation.

[3] Hajdinák, P., Czobor, Á., Lőrincz, T., Szarka, A. "The Problem of Glutathione Determination: a Comparative Study on the Measurement of Glutathione from Plant Cells", Periodica Polytechnica Chemical Engineering, pp. 1-9, 2018. https://doi.org/10.3311/PPch.11785

[4] Yuan, L., Kaplowitz, N. "Glutathione in liver diseases and hepatotoxicity", Molecular Aspects of Medicine, 30(1-2), pp. 29-41, 2009.

https://doi.org/10.1016/j.mam.2008.08.003 
[5] Lörincz, T., Jemnitz, K., Kardon, T., Mandl, J., Szarka, A. "Ferroptosis is Involved in Acetaminophen Induced Cell Death", Pathology \& Oncology Research, 21(4), pp. 1115-1121, 2015. https://doi.org/10.1007/s12253-015-9946-3

[6] Xie, Y., Hou, W., Song, X., Yu, Y., Huang, J., Sun, X., Kang, R., Tang, D. "Ferroptosis: process and function", Cell Death \& Differentiation, 23(3), pp. 369-379, 2016. https://doi.org/10.1038/cdd.2015.158

[7] Plačková, P., Šála, M., Šmídková, M., Dejmek, M., Hřebabecký, H., Nencka, R., Thibaut, H.-J., Neyts, J., Mertlikova-Kaiserova, H. "9-Norbornyl-6-chloropurine (NCP) induces cell death through GSH depletion-associated ER stress and mitochondrial dysfunction", Free Radical Biology and Medicine, 97, pp. 223-235, 2016. https://doi.org/10.1016/j.freeradbiomed.2016.06.004

[8] Mancilla, H., Maldonado, R., Cereceda, K., Villarroel-Espíndola, F., Montes De Oca, M., Angulo, C., Castro, M. A., Slebe, J. C., Vera, J. C., Lavandero, S., Concha, I. I. "Glutathione Depletion Induces Spermatogonial Cell Autophagy", Journal of Cellular Biochemistry, 116(10), pp. 2283-2292, 2015. https://doi.org/10.1002/jcb.25178

[9] Dolma, S., Lessnick, S. L., Hahn, W. C., Stockwell, B. R. "Identification of genotype-selective antitumor agents using synthetic lethal chemical screening in engineered human tumor cells", Cancer Cell, 3(3), pp. 285-296, 2003. https://doi.org/10.1016/S1535-6108(03)00050-3

[10] Dixon, S. J., Lemberg, K. M., Lamprecht, M. R., Skouta, R., Zaitsev, E. M., Gleason, C. E., Patel, D. N., Bauer, A. J., Cantley, A. M., Yang, W. S., Morrison, B., Stockwell, B. R. "Ferroptosis: An Iron-Dependent Form of Nonapoptotic Cell Death", Cell, 149(5), pp. 1060-1072, 2012. https://doi.org/10.1016/j.cel1.2012.03.042

[11] Yang, W. S., SriRamaratnam, R., Welsch, M. E., Shimada, K., Skouta, R., Viswanathan, V. S., Cheah, J. H., Clemons, P. A., Shamji, A. F., Clish, C. B., Brown, L. M., Girotti, A. W., Cornish, V. W., Schreiber, S. L., Stockwell, B. R. "Regulation of Ferroptotic Cancer Cell Death by GPX4", Cell, 156(1-2), pp. 317331. 2014.

https://doi.org/10.1016/j.cell.2013.12.010

[12] Friedmann Angeli, J. P., Schneider, M., Proneth, B., Tyurina, Y. Y., Tyurin, V. A., Hammond, V. J., Herbach, N., Aichler, M., Walch, A., Eggenhofer, E., Basavarajappa, D., Rådmark, O., Kobayashi, S., Seibt, T., Beck, H., Neff, F., Esposito, I., Wanke, R., Förster, H., Yefremova, O., Heinrichmeyer, M., Bornkamm, G. W., Geissler, E. K., Thomas, S. B., Stockwell, B. R., O’Donnell, V. B., Kagan, V. E., Schick, J. A., Conrad, M. „Inactivation of the ferroptosis regulator Gpx4 triggers acute renal failure in mice", Nature Cell Biology, 16(12), pp. 1180-1191, 2014. https://doi.org/10.1038/ncb3064

[13] Shimada, K., Skouta, R., Kaplan, A., Yang, W. S., Hayano, M., Dixon, S. J., Brown, L. M., Valenzuela, C. A., Wolpaw, A. J., Stockwell, B. R. "Global survey of cell death mechanisms reveals metabolic regulation of ferroptosis", Nature Chemical Biology, 12(7), pp. 497-503, 2016.

https://doi.org/10.1038/nchembio.2079
[14] Gaschler, M. M., Andia, A. A., Liu, H., Csuka, J. M., Hurlocker, B., Vaiana, C. A., Heindel, D. W., Zuckerman, D. S., Bos, P. H., Reznik, E., Ye, L. F., Tyurina, Y. Y., Lin, A. J., Shchepinov, M. S., Chan, A. Y., Peguero-Pereira, E., Fomich, M. A., Daniels, J. D., Bekish, A. V., Shmanai, V. V., Kagan, V. E., Mahal, L. K., Woerpel, K. A., Stockwell, B. R. "FINO2 initiates ferroptosis through GPX4 inactivation and iron oxidation", Nature Chemical Biology, 14(5), pp. 507-515, 2018.

https://doi.org/10.1038/s41589-018-0031-6

[15] Arai, M., Imai, H., Sumi, D., Imanaka, T., Takano, T., Chiba, N., Nakagawa, Y. "Import into Mitochondria of Phospholipid Hydroperoxide Glutathione Peroxidase requires a Leader Sequence", Biochemical and Biophysical Research Communications, 227(2), pp. 433-439, 1996.

https://doi.org/10.1006/bbrc.1996.1525

[16] Savaskan, N. E., Ufer, C., Kühn, H., Borchert, A. "Molecular biology of glutathione peroxidase 4: from genomic structure to developmental expression and neural function", Biological Chemistry, 388(10), pp. 1007-1017, 2007. https://doi.org/10.1515/BC.2007.126

[17] Liang, H., Yoo, S. E., Na, R., Walter, C. A., Richardson, A., Ran, Q. "Short Form Glutathione Peroxidase 4 Is the Essential Isoform Required for Survival and Somatic Mitochondrial Functions", The Journal of Biological Chemistry, 284(45), pp. 30836-30844, 2009. https://doi.org/10.1074/jbc.M109.032839

[18] Pfeifer, H., Conrad, M., Roethlein, D., Kyriakopoulos, A., Brielmeier, M., Bornkamm, G. W., Behne, D. "Identification of a specific sperm nuclei selenoenzyme necessary for protamine thiol cross-linking during sperm maturation", The FASEB Journal, 15(7), pp. 1236-1238, 2001. https://doi.org/10.1096/fj.00-0655fje

[19] Conrad, M., Moreno, S. G., Sinowatz, F., Ursini, F., Kölle, S., Roveri, A., Brielmeier, M., Wurst, W., Maiorino, M., Bornkamm, G. W. "The Nuclear Form of Phospholipid Hydroperoxide Glutathione Peroxidase Is a Protein Thiol Peroxidase Contributing to Sperm Chromatin Stability", Molecular and Cellular Biology, 25(17), pp. 7637-7644, 2005. https://doi.org/10.1128/MCB.25.17.7637-7644.2005

[20] Rigoulet, M., Yoboue, E. D., Devin, A. „Mitochondrial ROS Generation and Its Regulation: Mechanisms Involved in $\mathrm{H}_{2} \mathrm{O}_{2}$ Signaling", Antioxidants \& Redox Signaling, 14(3), pp. 459-468, 2011.

https://doi.org/10.1089/ars.2010.3363

[21] Yang, W. S., Stockwell, B. R. "Synthetic Lethal Screening Identifies Compounds Activating Iron-Dependent, Nonapoptotic Cell Death in Oncogenic-RAS-Harboring Cancer Cells", Chemistry and Biology, 15(3), pp. 234-245, 2008. https://doi.org/10.1016/j.chembiol.2008.02.010

[22] Jelinek, A., Heyder, L., Daude, M., Plessner, M., Krippner, S., Grosse, R., Diederich, W. E., Culmsee, C. "Mitochondrial rescue prevents glutathione peroxidase-dependent ferroptosis", Free Radical Biology and Medicine, 117, pp. 45-57, 2018. https://doi.org/10.1016/j.freeradbiomed.2018.01.019 
[23] Skouta, R., Dixon, S. J., Wang, J., Dunn, D. E., Orman, M., Shimada, K., Rosenberg, P. A., Lo, D. C., Weinberg, J. M., Linkermann, A., Stockwell, B. R. "Ferrostatins Inhibit Oxidative Lipid Damage and Cell Death in Diverse Disease Models", Journal of the American Chemical Society, 136(12), pp. 4551-4556, 2014. https://doi.org/10.1021/ja411006a

[24] Baulies, A., Montero, J., Matías, N., Insausti, N., Terrones, O., Basañez, G., Vallejo, C., Conde de La Rosa, L., Martinez, L., Robles, D., Morales, A., Abian, J., Carrascal, M., Machida, K., Kumar, D. B. U., Tsukamoto, H., Kaplowitz, N., Garcia-Ruiz, C., Fernández-Checa, J. C. "The 2-oxoglutarate carrier promotes liver cancer by sustaining mitochondrial GSH despite cholesterol loading", Redox Biology, 14, pp. 164-177, 2018.

https://doi.org/10.1016/j.redox.2017.08.022

[25] Emanuelsson, O., Nielsen, H., Brunak, S., Von Heijne, G. "Predicting Subcellular Localization of Proteins Based on their N-terminal Amino Acid Sequence", Journal of Molecular Biology, 300(4), pp. 1005-1016, 2000.

https://doi.org/10.1006/jmbi.2000.3903

[26] Claros, M. G., Vincens, P. "Computational Method to Predict Mitochondrially Imported Proteins and their Targeting Sequences", European Journal of Biochemistry, 241(3), pp. 779-786, 1996. https://doi.org/10.1111/j.1432-1033.1996.00779.x

[27] Small, I., Peeters, N., Legeai, F., Lurin, C. "Predotar: A tool for rapidly screening proteomes for $\mathrm{N}$-terminal targeting sequences", Proteomics, 4(6), pp. 1581-1590, 2004. https://doi.org/10.1002/pmic.200300776

[28] Nakai, K., Horton, P. "PSORT: a program for detecting sorting signals in proteins and predicting their subcellular localization", Trends in Biochemical Sciences, 24(1), pp. 34-35, 1999. https://doi.org/10.1016/S0968-0004(98)01336-X

[29] Bannai, H., Tamada, Y., Maruyama, O., Nakai, K., Miyano, S. "Extensive feature detection of N-terminal protein sorting signals", Bioinformatics, 18(2), pp. 298-305, 2002. https://doi.org/10.1093/bioinformatics/18.2.298

[30] Höglund, A., Dönnes, P., Blum, T., Adolph, H.-W., Kohlbacher, O. "MultiLoc: prediction of protein subcellular localization using $\mathrm{N}$-terminal targeting sequences, sequence motifs and amino acid composition", Bioinformatics, 22(10), pp. 1158-1165, 2006. https://doi.org/10.1093/bioinformatics/bt1002

[31] King, B. R., Vural, S., Pandey, S., Barteau, A., Guda, C. "ngLOC: software and web server for predicting protein subcellular localization in prokaryotes and eukaryotes", BMC Research Notes, 5(1), pp. 351, 2012.

https://doi.org/10.1186/1756-0500-5-351

[32] Briesemeister, S., Rahnenführer, J., Kohlbacher, O. "YLoc-an interpretable web server for predicting subcellular localization.”. Nucleic Acids Research, 38(suppl_2), pp. W497-W502, 2010. https://doi.org/10.1093/nar/gkq477

[33] Yu, C.-S., Chen, Y.-C., Lu, C.-H., Hwang, J.-K. "Prediction of protein subcellular localization", Proteins, 64(3), pp. 643-651, 2006. https://doi.org/10.1002/prot.21018
[34] Balogh, T., Lörincz, T., Stiller, I., Mandl, J., Bánhegyi, G., Szarka, A. "The Level of ALR is Regulated by the Quantity of Mitochondrial DNA", Pathology \& Oncology Research, 22(2), pp. 431-437, 2016. https://doi.org/10.1007/s12253-015-0020-y

[35] Czobor, Á., Hajdinák, P., Szarka, A. "Rapid ascorbate response to bacterial elicitor treatment in Arabidopsis thaliana cells", Acta Physiologiae Plantarum, 39(2), pp. 62, 2017. https://doi.org/10.1007/s11738-017-2365-1

[36] Robin, M.-A., Prabu, S. K., Raza, H., Anandatheerthavarada, H. K., Avadhani, N. G. "Phosphorylation Enhances Mitochondrial Targeting of GSTA4-4 through Increased Affinity for Binding to Cytoplasmic Hsp70", The Journal of Biological Chemistry, 278(21), pp. 18960-18970, 2003. https://doi.org/10.1074/jbc.M301807200

[37] Scheerer, P., Borchert, A., Krauss, N., Wessner, H., Gerth, C., Höhne, W., Kuhn, H. "Structural Basis for Catalytic Activity and Enzyme Polymerization of Phospholipid Hydroperoxide Glutathione Peroxidase-4 (GPx4)", Biochemistry, 46(31), pp. 9041-9049, 2007. https://oi.org/10.1021/bi700840d

[38] Knockaert, L., Fromenty, B., Robin, M.-A. "Mechanisms of mitochondrial targeting of cytochrome P450 2E1: Physiopathological role in liver injury and obesity", The FEBS Journal, 278(22), pp. 4252-4260, 2011. https://doi.org/10.1111/j.1742-4658.2011.08357.x

[39] Sangar, M. C., Bansal, S., Avadhani, N. G. "Bimodal targeting of microsomal cytochrome P450s to mitochondria: implications in drug metabolism and toxicity", Expert Opinion on Drug Metabolism \& Toxicology, 6(10), pp. 1231-1251, 2010. https://doi.org/10.1517/17425255.2010.503955

[40] Anandatheerthavarada, H. K., Sepuri, N. B. V., Avadhani, N. G. "Mitochondrial Targeting of Cytochrome P450 Proteins Containing $\mathrm{NH}_{2}$-terminal Chimeric Signals Involves an Unusual TOM20/TOM22 Bypass Mechanism", The Journal of Biological Chemistry, 284(25), pp. 17352-17363, 2009. https://doi.org/10.1074/jbc.M109.007492

[41] Su, P., Rennert, H., Shayiq, R. M., Yamamoto, R., Zheng, Y.-M., Addya, S., Strauss, J. F., Avadhani, N. G. "A cDNA Encoding a Rat Mitochondrial Cytochrome P450 Catalyzing Both the 26-Hydroxylation of Cholesterol and 25-Hydroxylation of Vitamin $\mathrm{D}_{3}$ : Gonadotropic Regulation of theCognate mRNA in Ovaries", DNA and Cell Biology, 9(9), pp. 657-665, 1990. https://doi.org/10.1089/dna.1990.9.657

[42] Kurosawa, K., Hayashi, N., Sato, N., Kamada, T., Tagawa, K. "Transport of glutathione across the mitochondrial membranes", Biochemical and Biophysical Research Communications, 167(1), pp. 367-372, 1990. https://doi.org/10.1016/0006-291X(90)91774-M

[43] Mårtensson, J., Lai, J. C., Meister, A. "High-affinity transport of glutathione is part of a multicomponent system essential for mitochondrial function", Proceedings of the National Academy of Sciences, 87(18), pp. 7185-7189, 1990. https://doi.org/10.1073/pnas.87.18.7185 
[44] Iacobazzi, V., Infantino, V., Castegna, A., Menga, A., Palmieri, E. M., Convertini, P., Palmieri, F. "Mitochondrial carriers in inflammation induced by bacterial endotoxin and cytokines", Biological Chemistry, 398(3), pp. 303-317, 2017.

https://doi.org/10.1515/hsz-2016-0260

[45] Booty, L. M., King, M. S., Thangaratnarajah, C., Majd, H., James, A. M., Kunji, E. R. S., Murphy, M. P. "The mitochondrial dicarboxylate and 2-oxoglutarate carriers do not transport glutathione", The FEBS Letters, 589(5), pp. 621-628, 2015.

https://doi.org/10.1016/j.febslet.2015.01.027
[46] Wilkins, H. M., Marquardt, K., Lash, L. H., Linseman, D. A. "Bcl-2 is a novel interacting partner for the 2-oxoglutarate carrier and a key regulator of mitochondrial glutathione", Free Radical Biology and Medicine, 52(2), pp. 410-419, 2012.

https://doi.org/10.1016/j.freeradbiomed.2011.10.495 